\title{
Subpopulation Differences in the Cardiovascular Efficacy of Long-Acting Glucagon-Like Peptide 1 Receptor Agonists in Type 2 Diabetes Mellitus: A Systematic Review and Meta-analysis
}

Liyun He $\cdot \mathrm{Na}$ Yang $\cdot$ Lingling Xu - Fan Ping $\cdot$ Wei Li

Yuxiu Li · Huabing Zhang (D)

Received: June 1, 2020 / Published online: July 30, 2020

(C) The Author(s) 2020

\begin{abstract}
Introduction: The cardiovascular efficacy of glucagon-like peptide 1 receptor agonists (GLP1RAs) in type 2 diabetes mellitus (T2DM) are well documented; however, the differences in cardiovascular efficacy among subpopulations remain unknown. This systematic review and meta-analysis aimed to explore the differences in cardiovascular efficacy of long-acting GLP1RAs among subpopulations of patients with T2DM and to assess the drug safety.

Methods: Relevant studies up to March 31, 2020 were searched for in six electronic databases, namely PubMed, Cochrane Library, Embase, Clinical Trials, Science Direct, and Web of Science. The primary outcome was threepoint major adverse cardiovascular events
\end{abstract}

Digital Features To view digital features for this article go to https://doi.org/10.6084/m9.figshare.12630125.

Electronic supplementary material The online version of this article (https://doi.org/10.1007/s13300020-00882-2) contains supplementary material, which is available to authorized users.

L. He $\cdot$ N. Yang $\cdot$ L. Xu - F. Ping - W. Li · Y. Li .

H. Zhang ( $\varangle)$

Department of Endocrinology, Key Laboratory of Endocrinology, Ministry of Health, Peking Union Medical College Hospital, Chinese Academy of Medical Sciences and Peking Union Medical

College, Beijing, China

e-mail: huabingzhangchn@163.com (including cardiovascular mortality, non-fatal myocardial infarction, and non-fatal stroke). Subpopulations were defined using ten selected influential factors, and the differences in cardiovascular efficacy in subpopulations stratified by different influential factors were accessed by synthesizing studies with random-effects models one by one.

Results: A total of six cardiovascular outcome trials of long-acting GLP-1RAs, comprising 49,936 participants, were included. Among stratified subpopulations, no significant differences in the cardiovascular efficacy of longacting GLP-1RAs were observed across the ten characteristics of subjects (all $P$ for interaction $>0.05$ ). Favorable trends were observed in the subpopulation with established cardiovascular disease (CVD) compared to that without $(P=0.171)$. With regards to safety, long-acting GLP-1RAs did not significantly increase the risk of retinopathy (OR 1.09; 95\% CI 0.92-1.29; $P=0.316)$, but increase the risk of serious gastrointestinal events (OR 1.37; 95\% CI 1.02-1.83; $P=0.037)$. Long-acting GLP-1RAs did not significantly increase the risk of serious adverse events (OR 0.92; 95\% CI 0.85-1.00; $P=0.039$ ).

Conclusions: Our analysis suggested no subpopulation differences in the cardiovascular efficacy of long-acting GLP-1RAs among stratified subpopulations, and favorable trends were only observed in the subpopulation with established CVD. These findings may have implications for the management of long-acting 
GLP-1RAs across subpopulations of patients with T2DM.

Keywords: Cardiovascular outcome; Longacting GLP-1 receptor agonists; Meta-analysis; Type 2 diabetes; Safety profiles; Subpopulation differences

\section{Key Summary Points}

Why carry out this study?

Many studies reported remarkable cardiovascular efficacy of glucagon-like peptide 1 receptor agonists (GLP-1RAs) in type 2 diabetes mellitus (T2DM), but the differences in cardiovascular efficacy among various subpopulations of patients with T2DM remained unknown.

What were the subpopulation differences in cardiovascular efficacy of long-acting GLP-1RAs among patients with T2DM?

\section{What was learned from the study?}

Our studies suggested that the subpopulation differences in cardiovascular efficacy of long-acting GLP1RAs were insignificant among subpopulations stratified by different influential factors, and only favorable trends were noticed in the subpopulation with established CVD.

Our findings might have implications for the management of long-acting GLP-1RAs treatments across various subpopulations of patients with T2DM, as well as the safety of long-acting GLP-1RAs.

\section{INTRODUCTION}

Cardiovascular disease (CVD) is the leading cause of death among patients with type 2 diabetes mellitus (T2DM), who are characterized as having a high risk for CVD [1]. As popular antidiabetic agents for patients with T2DM [2], glucagon-like peptide 1 receptor agonists (GLP1RAs) have attracted increased universal attention for their cardioprotective efficacy, which might be attributed to the pleiotropic effects of ameliorating cardiovascular risk factors [2, 3]. Long-acting GLP-1Ras have shown significant cardiovascular benefits in trials [4], while shortacting GLP-1RAs (lixisenatide) have not shown favorable efficacy in terms of cardiovascular outcomes [5]. This difference might result from the inadequate time of GLP-1R activation with lixisenatide $[2,6]$. In addition, diverse characteristics of participants, such as age, sex, and body mass index, might impact the cardiovascular efficacy of antidiabetic drugs. A recent study demonstrated significant differences in the cardiovascular benefits of sodium-glucose cotransporter 2 inhibitors (SGLT2i) within subpopulations stratified by atherosclerotic cardiovascular disease status [7]. However, as a result of the paucity of relevant investigations and analyses, influential factors and subpopulation differences in cardiovascular efficacy remain unknown and controversial. Exploring subpopulation differences and ascertaining the influence factors could help to optimize the treatments of long-acting GLP-1RAs for patients with T2DM. Intriguingly, subpopulations exhibiting greater cardiovascular efficacy from long-acting GLP-1RAs could be more appropriately recommended for the administration of long-acting GLP-1RAs. Moreover, no up-to-date systematic reviews and meta-analyses have explored subpopulation differences in the cardiovascular efficacy of long-acting GLP-1RAs. Hence, it is necessary to synthesize the results of recently published studies in order to explore the influential factors and subpopulation differences of the administration of long-acting GLP-1RAs.

To address this gap in knowledge, this systematic review and meta-analysis synthesized the randomized controlled cardiovascular outcome trials (CVOTs) of long-acting GLP-1RAs with the intention to explore the differences in cardiovascular efficacy among subpopulations of patients with T2DM, as well as to determine the overall cardiovascular safety of long-acting GLP-1RAs. 


\section{METHODS}

The present study adhered to the standards of the preferred reporting items for systematic review and meta-analysis (PRISMA) for a metaanalysis and systematic review of randomized clinical trials [8] (Supplementary Appendix). This meta-analysis and systematic review was prospectively registered in PROSPERO (CRD42019132137).

\section{Compliance with Ethics Guidelines}

This article is based on previously conducted studies and does not contain any studies with human participants or animals performed by any of the authors.

\section{Study Eligibility}

Inclusion criteria: (1) randomized controlled clinical trials (RCTs) performed in adults (age $\geq 18$ years) with T2DM, of any diabetes duration, geographical distribution, countriy, race, follow-up, sample, and any background treatment administrated to participants; (2) including at least one intervention group and one more control; (3) treatment with long-acting GLP-1RAs as the intervention groups; and (4) designed to evaluate the cardiovascular outcome of GLP-1RAs, such as three-point major adverse cardiovascular events (MACE; cardiovascular death, non-fatal myocardial infarction, or non-fatal stroke) and four-point MACE (including cardiovascular death, myocardial infarction, stroke, or hospitalization for unstable angina death) for the primary outcome.

Exclusion criteria: (1) non-human studies, such as animal studies; (2) studies with no results of cardiovascular outcomes; (3) studies with insufficient data (without data of subpopulation analysis) or with incomplete trials; and (4) reviews, editorials, commentaries, opinion articles, or conference abstracts without original data.

\section{Study Identification and Selection}

PubMed, Cochrane Library, Embase, Clinical Trials, Science Direct, and all databases in Web of Science were scrupulously searched up to March 31, 2020 without limits of language. Full search strategies are listed in the Supplementary Appendix. Two reviewers ( $\mathrm{LH}$ and $\mathrm{HZ}$ ) independently conducted systematic literature retrievals for RCTs, and disagreements were settled through discussion with a third reviewer. The major search terms and were as follows: "diabetes mellitus, diabetes," "GLP-1 receptor agonists, exenatide, liraglutide, semaglutide, albiglutide, dulaglutide, taspoglutide," and "cardiovascular outcome, cardiovascular events, cardiovascular diseases, major adverse cardiovascular events, MACE."

On the basis of inclusion and exclusion criteria, two reviewers (LH and NY) screened the titles and abstracts of the retrieved literatures independently. The selection process was repeated twice by each reviewer. The references of the identified literatures were manually searched and screened as an important supplement. Discrepancies were resolved through discussions with other team members until a consensus was reached.

\section{Data Extraction and Quality Assessment}

Data were extracted independently by two reviewers (LH and HZ), using standardized predefined data extraction forms. The primary efficacy outcome for this analysis was threepoint MACE (cardiovascular mortality, non-fatal myocardial infarction, non-fatal stroke), and cardiovascular mortality, non-fatal myocardial infarction, non-fatal stroke, all-cause mortality, and hospitalization for heart failure (HF) were also analyzed individually. Importantly, the characteristics of interest for the stratifications of subpopulations were as follows: age, sex, region, race, diabetes mellitus (DM) duration, history of HF, history of established CVD, body mass index (BMI), glycated hemoglobin levels, and estimated glomerular filtration rate (GFR). The safety endpoints comprised severe hypoglycemia, pancreatitis, pancreatic cancer, 
serious gastrointestinal events, retinopathy, adverse events (AEs) leading to drug discontinuation, and serious adverse events (SAE). The definition of primary safety endpoints is outlined in the Supplementary Appendix.

The quality of the enrolled RCTs was assessed with the Cochrane risk of bias assessment tool [9]. All trials met the criteria for being performed well, and had a low risk of bias according to the Cochrane tool for assessing risk of bias in randomized clinical trials (Supplementary Appendix).

\section{Statistical Analysis}

Hazard ratios (HRs) with 95\% confidence intervals (CIs) were synthesized for cardiovascular efficacy outcomes with random-effects models. Meta-regression analyses were performed using the residual maximum likelihood method and Hartung-Knapp adjustment for tests of interactions to assess the differences in cardiovascular efficacy across the subpopulations. Overall odds ratios (ORs) with 95\% CIs were applied for safety endpoints, which were obtained through inverse-variance weights for the weighted mean $\log \mathrm{OR}$ estimate, and then exponentiated. Heterogeneity was quantified with Cochrane $Q$ statistic and $I^{2}$ statistic, which was judged as low $\left(I^{2} \leq 25 \%\right)$, moderate $\left(25 \%<I^{2} \leq 50 \%\right)$, or high $\left(I^{2} \geq 75 \%\right)$. Statistical analyses were conducted with Stata statistical software version 15.0 (Stata Corp, College Station, TX, USA), and $P$ values less than 0.05 were considered statistically significant.

\section{RESULTS}

\section{Study Characteristics and Quality Assessment}

A total of six CVOTs of long-acting GLP-1RAs [10-15], with 49,936 participants, met the selection criteria in this meta-analysis. The study selection flowchart is shown in Fig. 1. The key design features of the trials and important baseline characteristics of the participants are listed in Table 1. Only the subjects in the
Harmony Outcomes trial were $100 \%$ with established CVD, while subjects in the REWIND trial were of the lowest proportion (34.3\%). Only the PIONEER-6 patients were orally administrated with long-acting GLP-1RAs. The REWIND trial had the longest follow-up time (5.4 years) and the lowest baseline glycated hemoglobin level (7.3\%). Participants in the SUSTAIN-6 trial possessed a high proportion of pre-existing retinopathy. The general baseline characteristics of all subjects were generally similar in all included trials (Table S1). All subjects were older than 50 years, with a mean age of 63.2 years, and women accounted for $38.5 \%$ of the total. Most of the participants were white, and were from Europe or North America (Table S1).

All included trials had a low risk of bias according to the Cochrane tool for assessing risk of bias in randomized clinical trials (Table S2).

\section{Overall Efficacy of Long-Acting GLP-1RAs}

The details of overall efficacy are shown in Table 2 and Fig. S1a-f. For three-point MACE, GLP-1RA treatment generated a significant relative risk reduction of $14 \%$ (HR 0.86 ; 95\% CI $0.82-0.91 ; \quad P<0.001)$ compared to placebo, similar to that observed for cardiovascular mortality (HR $0.86 ; \quad 95 \%$ CI $0.79-0.95$; $P=0.002)$. There were significant risk reductions in non-fatal myocardial infarction of $12 \%$ (HR 0.88; 95\% CI 0.80-0.98; $P=0.015$ ), nonfatal stroke of $18 \%$ (HR 0.82; 95\% CI 0.74-0.91; $P<0.001$ ), all-cause mortality of $12 \%$ (HR 0.88 ; 95\% CI 0.81-0.95; $P=0.001)$, and hospitalization for heart failure (HR 0.91; 95\% CI $0.83-0.99 ; P=0.036$ ) compared to placebo.

\section{Analyses of Subpopulation Differences}

The data and results of various subpopulations are shown in Tables 3, S3, and S4 and Figs. 2 and S2. There were no significant differences in the cardiovascular benefits among subpopulations classified by various factors during the treatments with long-acting GLP-1RAs (all $P$ for interaction $>0.05$ ) (Table 3, Figs. 2 and S2). The subgroup with previously established CVD 


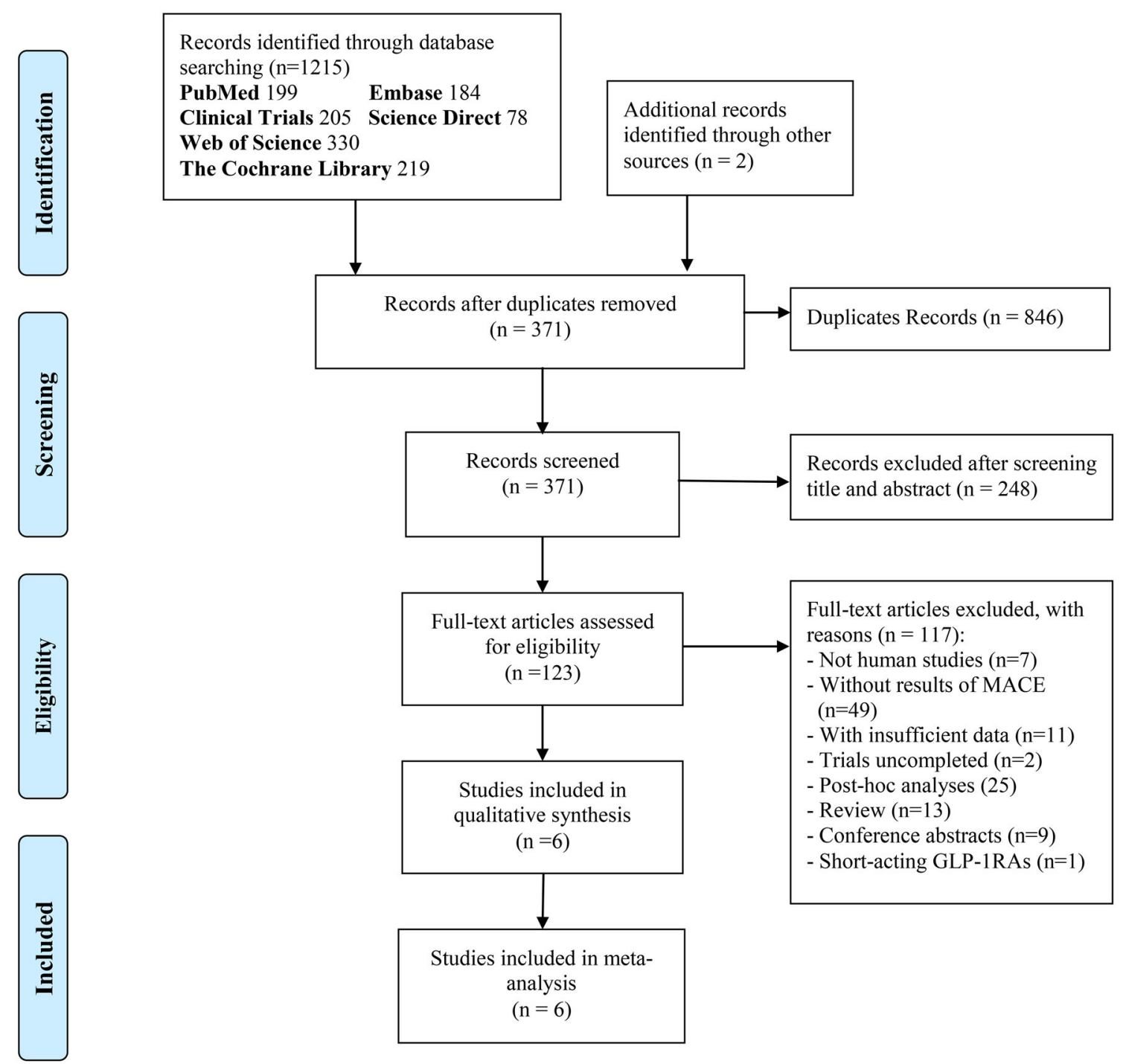

Fig. 1 Flow chart of search strategy. MACE major adverse cardiovascular events

showed a clear tendency for greater favorable cardioprotective effects compared to those without established CVD [HR (95\% CI) 0.84 $(0.80,0.89)$ vs. $0.94(0.82,1.07) ; P$ for interaction $=0.171]$ (Fig. 3), the comparison of which was carried out on the basis of the HRs of the two groups. In the placebo group without established CVD, the incidence of three-point MACE per year was mostly below $1.9 \%$ /year, except $2.42 \% /$ year in the PIONEER-6 trial (Table S4). Similarly, Asian populations exhibited a slightly favorable tendency for greater cardiovascular benefits in the stratification of race $(P$ for interaction $=0.148) \quad($ Fig. 4$)$. The subpopulation with baseline $\mathrm{BMI}<30 \mathrm{~kg} / \mathrm{m}^{2}$ showed moderate heterogeneity $\left(I^{2}=52.3 \%\right)$ (Fig. 5), while no heterogeneity was observed in the subpopulation characterized with baseline $\mathrm{BMI} \geq 30 \mathrm{~kg} / \mathrm{m}^{2}\left(I^{2}=0 \%\right)$ (Fig. 5 ).

\section{Safety Outcomes}

With regards to the safety endpoints (Table 4, Fig. S3), there were no significant increases in the risk of severe hypoglycemia (OR $0.95 ; 95 \%$ CI 0.77-1.16; $P=0.597$ ), pancreatitis (OR 0.92; 95\% CI $0.63-1.34 ; P=0.657)$, and pancreatic 


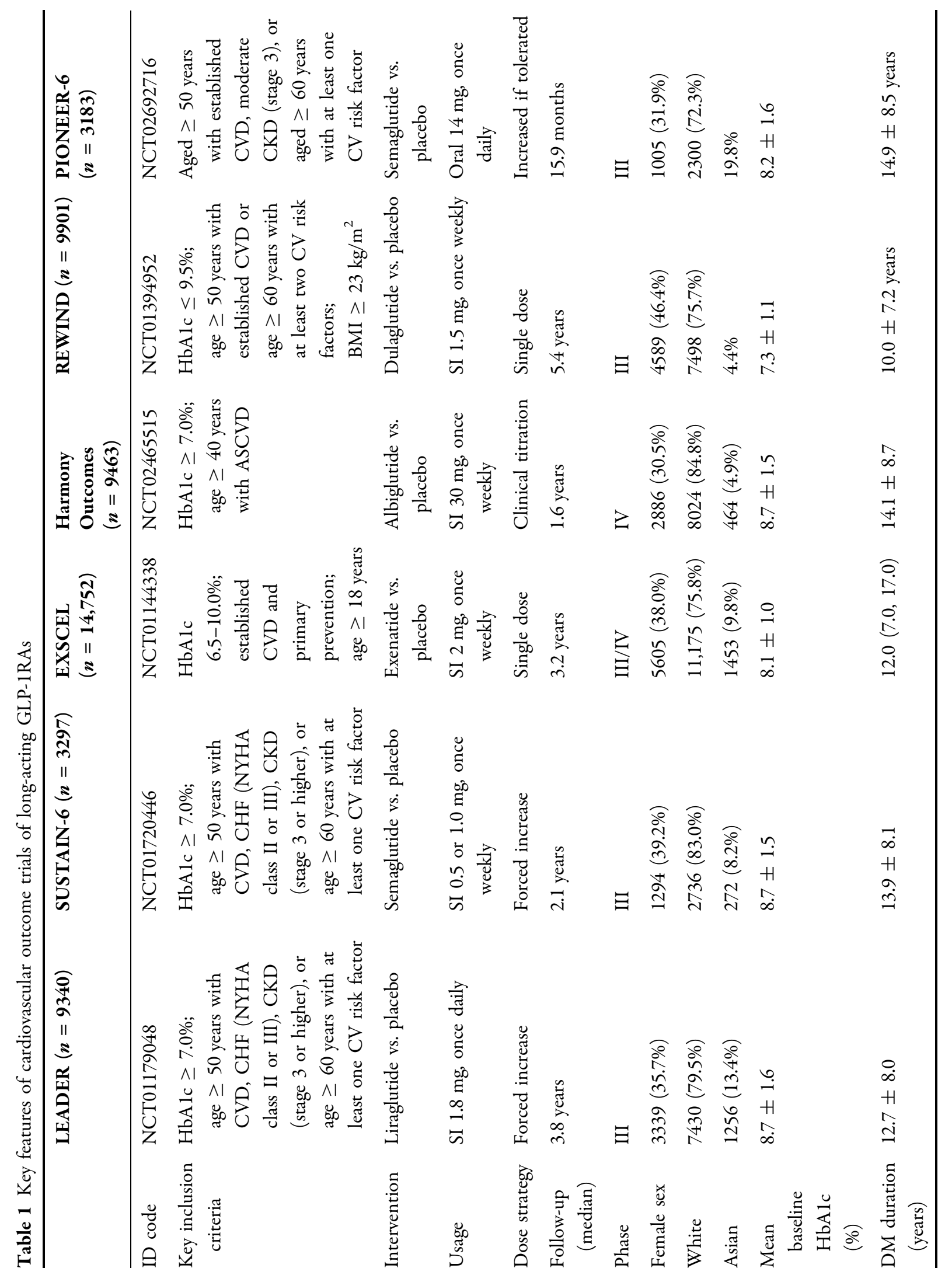




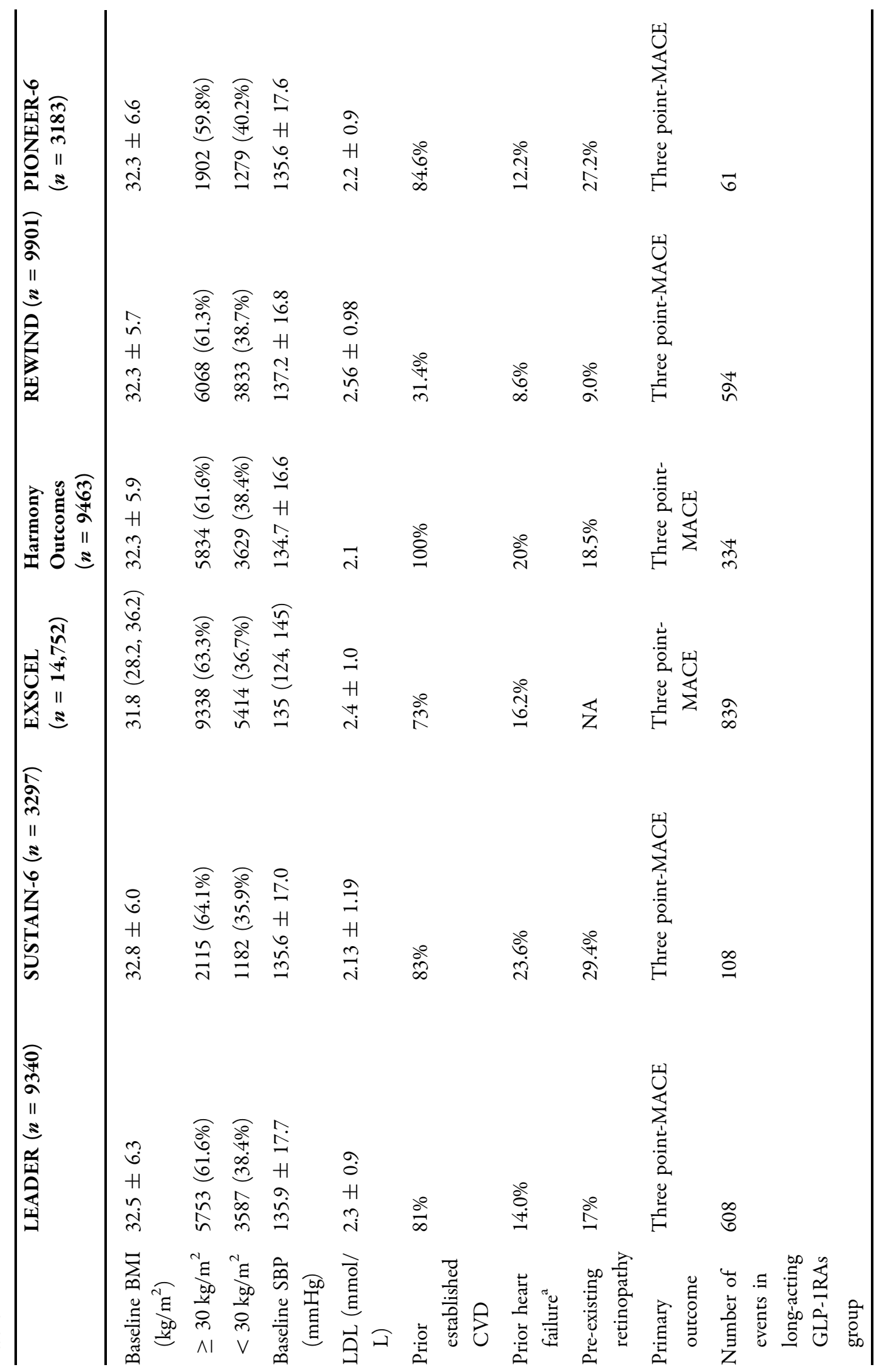




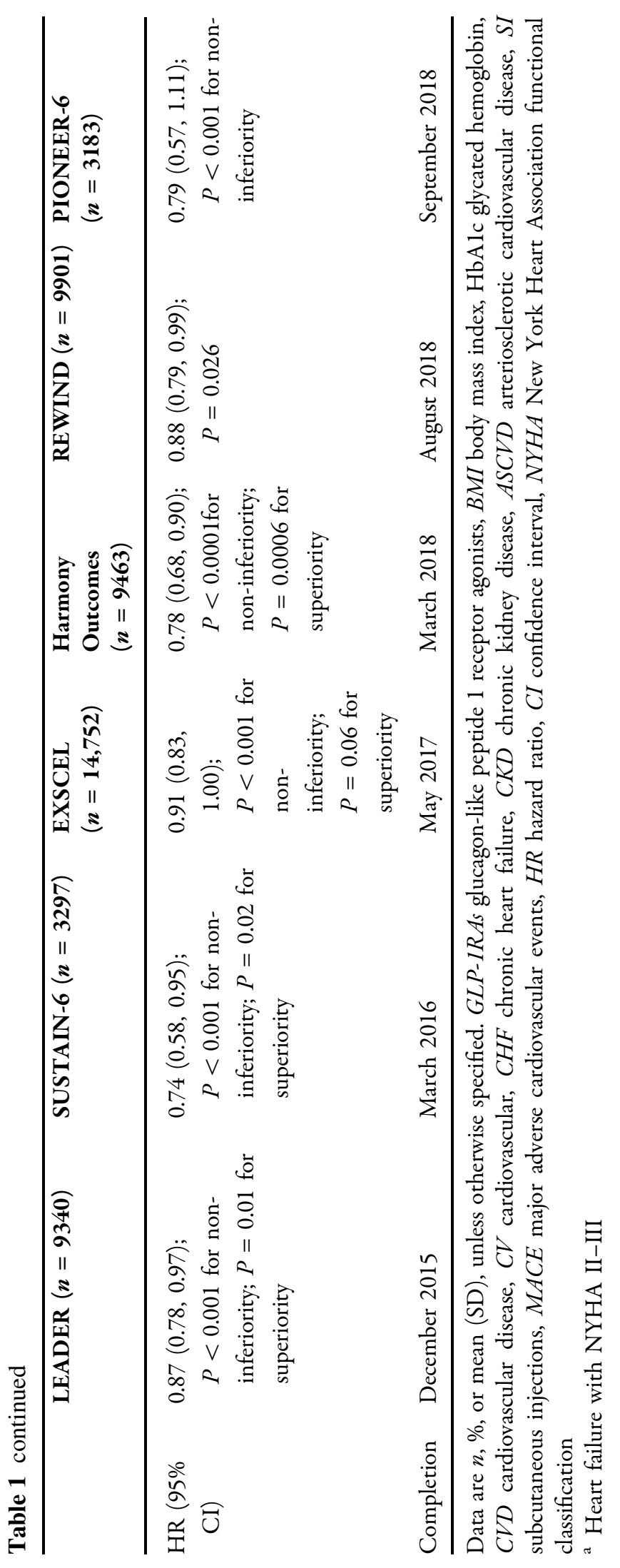




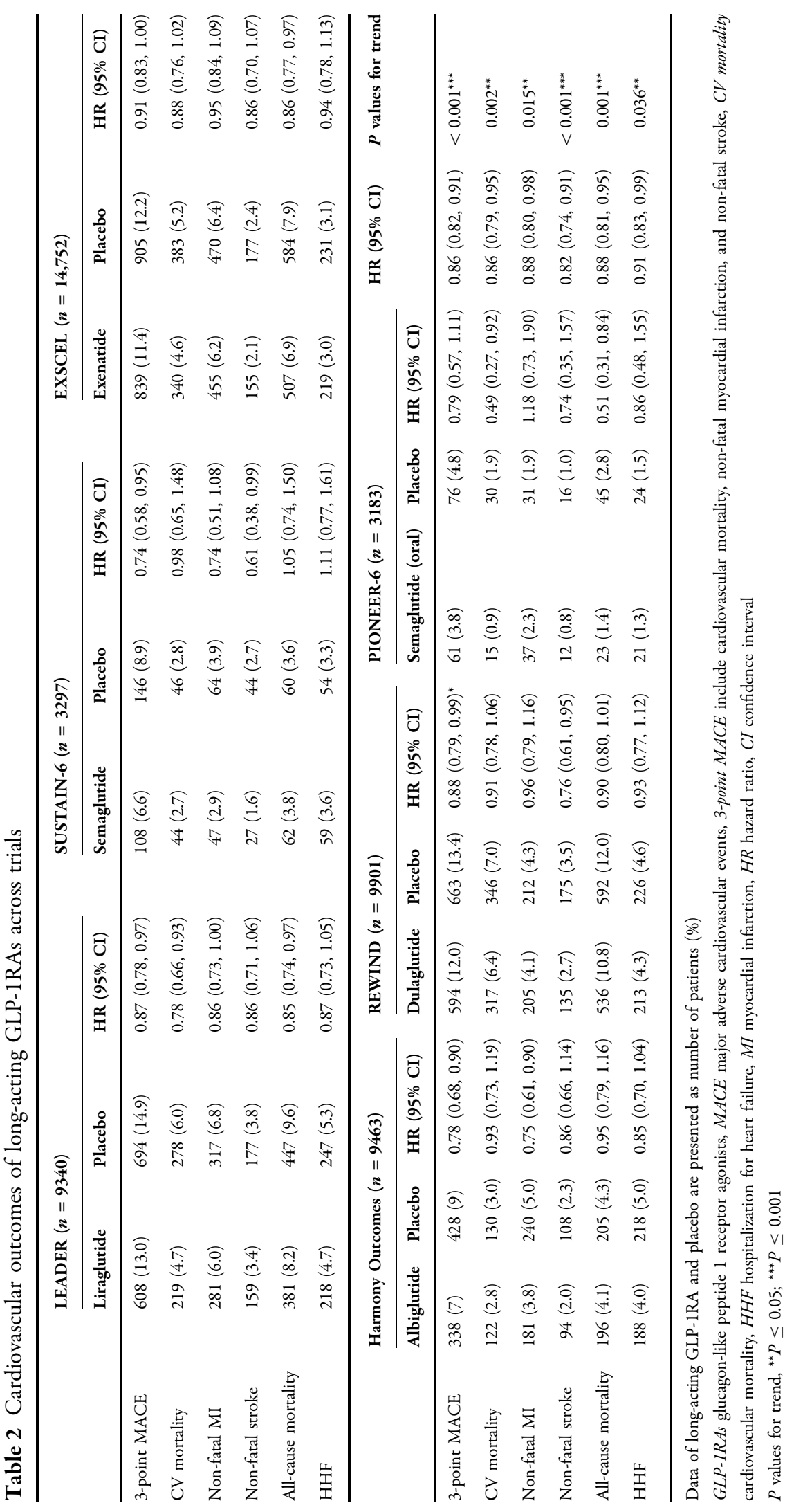




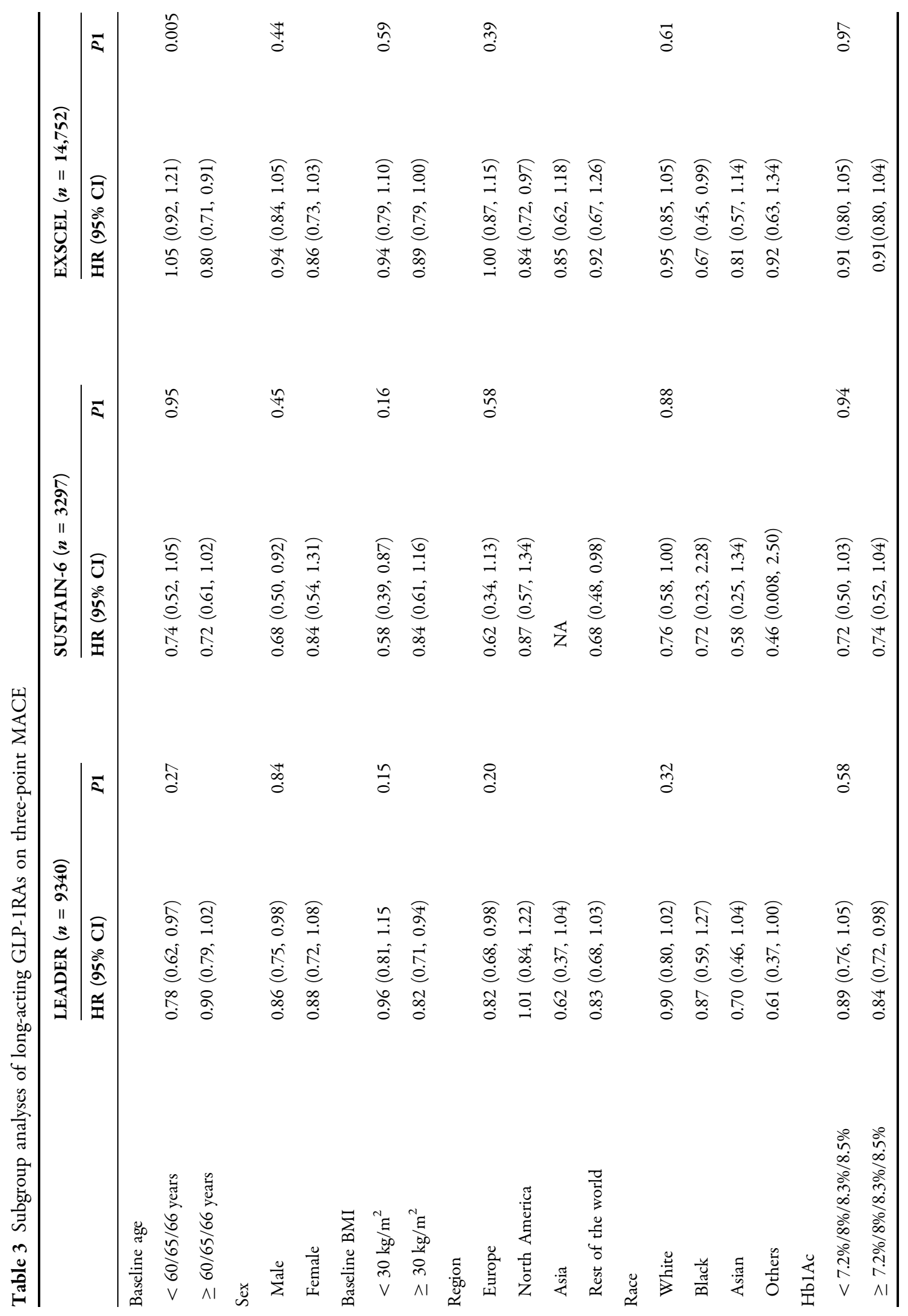




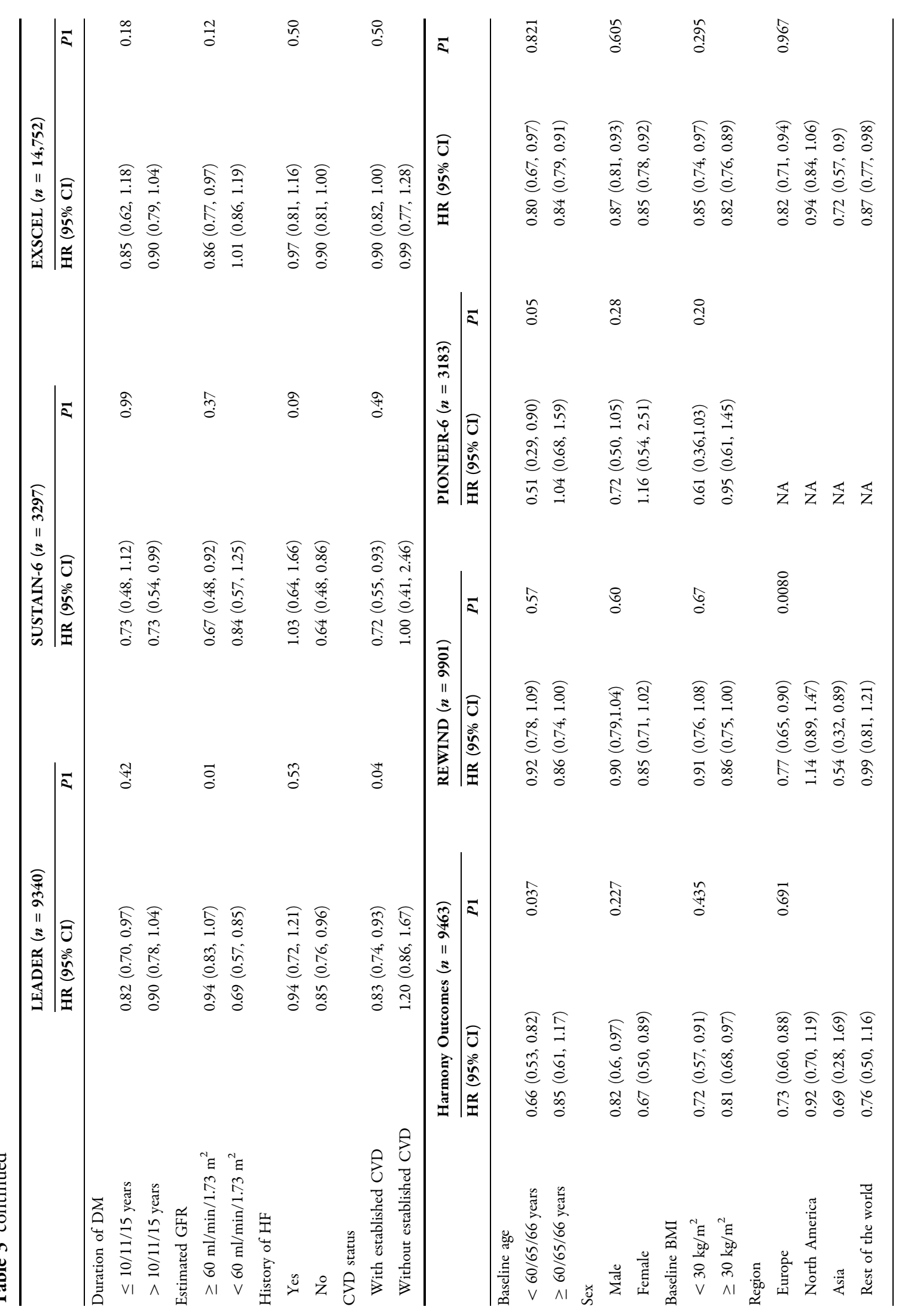




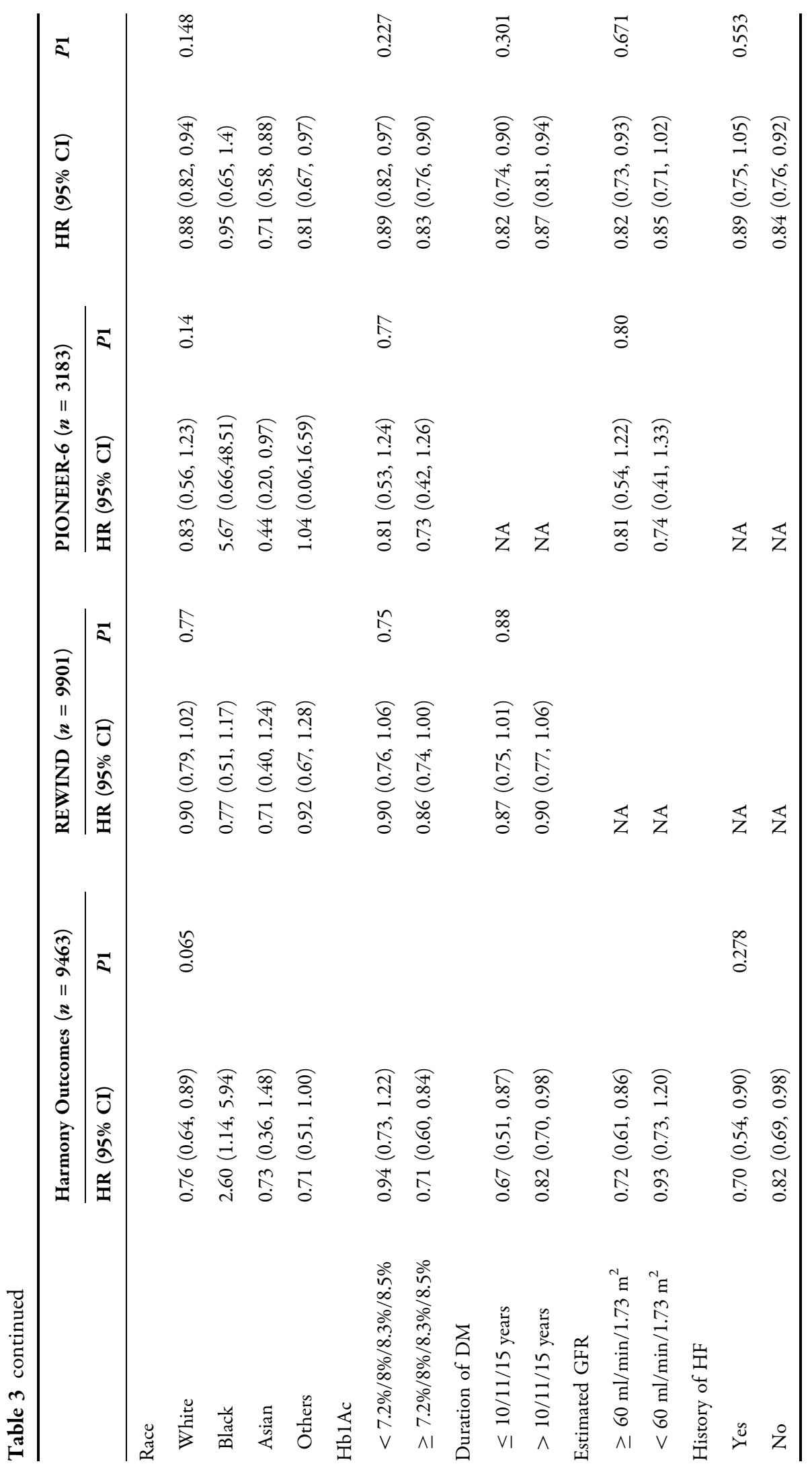




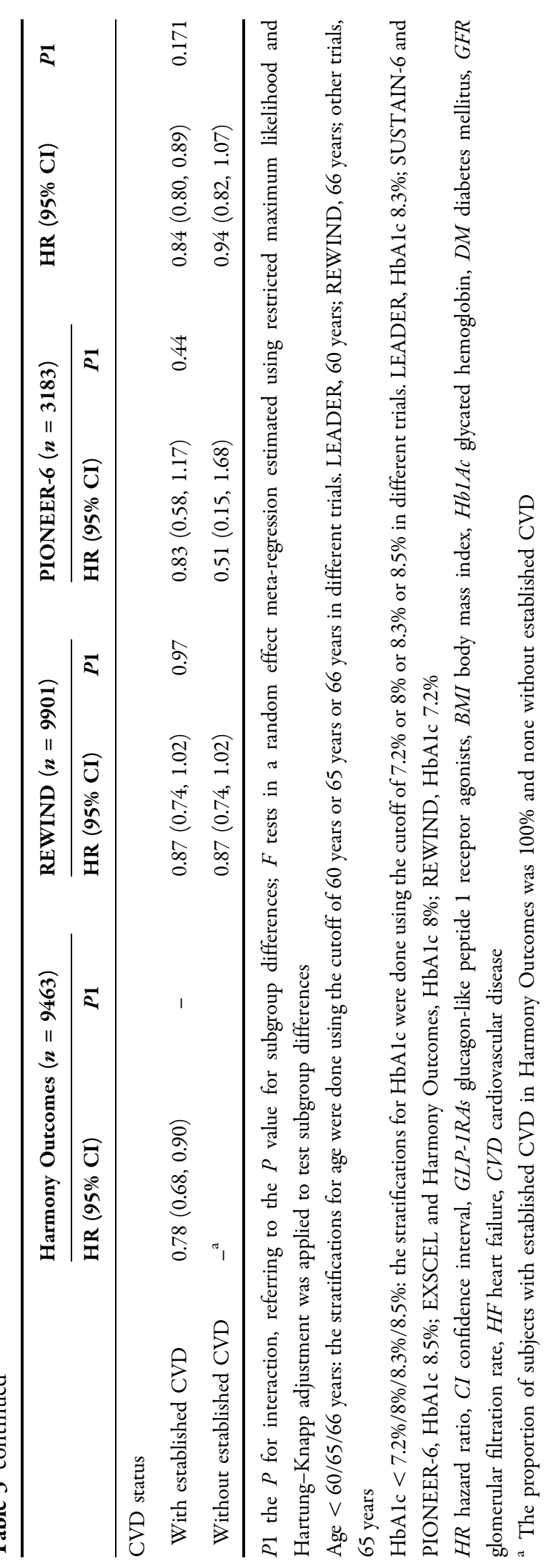




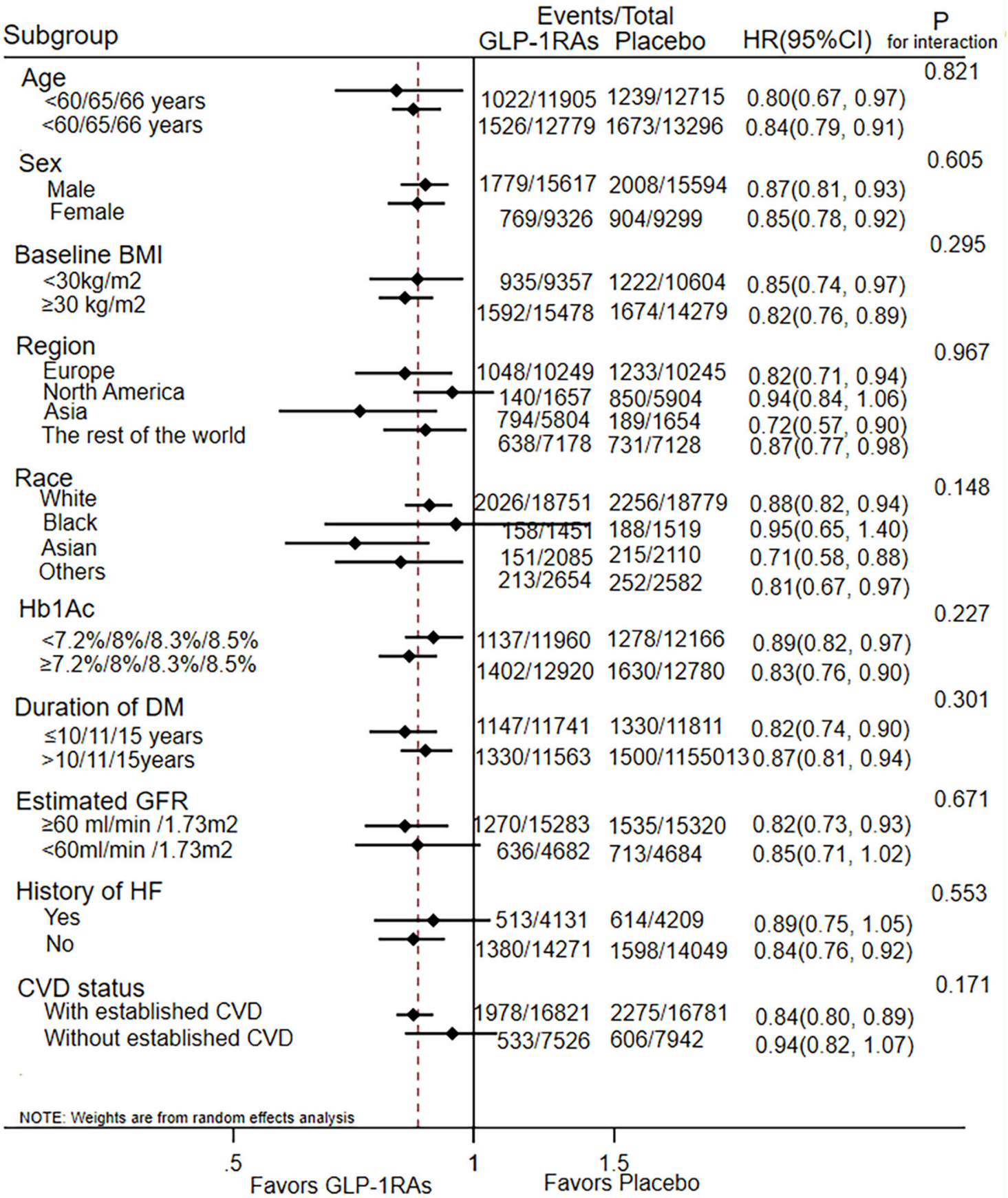

Fig. 2 Forest plot of subgroup analyses. $P$ for interaction: the $P$ value for differences within subgroups. Age $<60 /$ 65/66 years: the stratifications for age were done using the cutoff of 60 years or 65 years or 66 years in different trials. LEADER, 60 years; REWIND, 66 years; other trials, 65 years. HbAlc $<7.2 \% / 8 \% / 8.3 \% / 8.5 \%$ : the stratifications for $\mathrm{HbAlc}$ were done using the cutoff of $7.2 \%$ or $8 \%$ or $8.3 \%$ or $8.5 \%$ in different trials. LEADER, HbAlc
8.3\%; SUSTAIN-6 and PIONEER-6, HbAlc 8.5\%; EXSCEL and Harmony Outcomes, HbAlc 8\%; REWIND, HbAlc 7.2\%. HR hazard ratio, CI confidence interval, GLP-1RAs glucagon-like peptide 1 receptor agonists, $D M$ diabetes mellitus, $G F R$ glomerular filtration rate, GFR glomerular filtration rate, $H F$ heart failure, $C V D$ cardiovascular disease 
CVD status

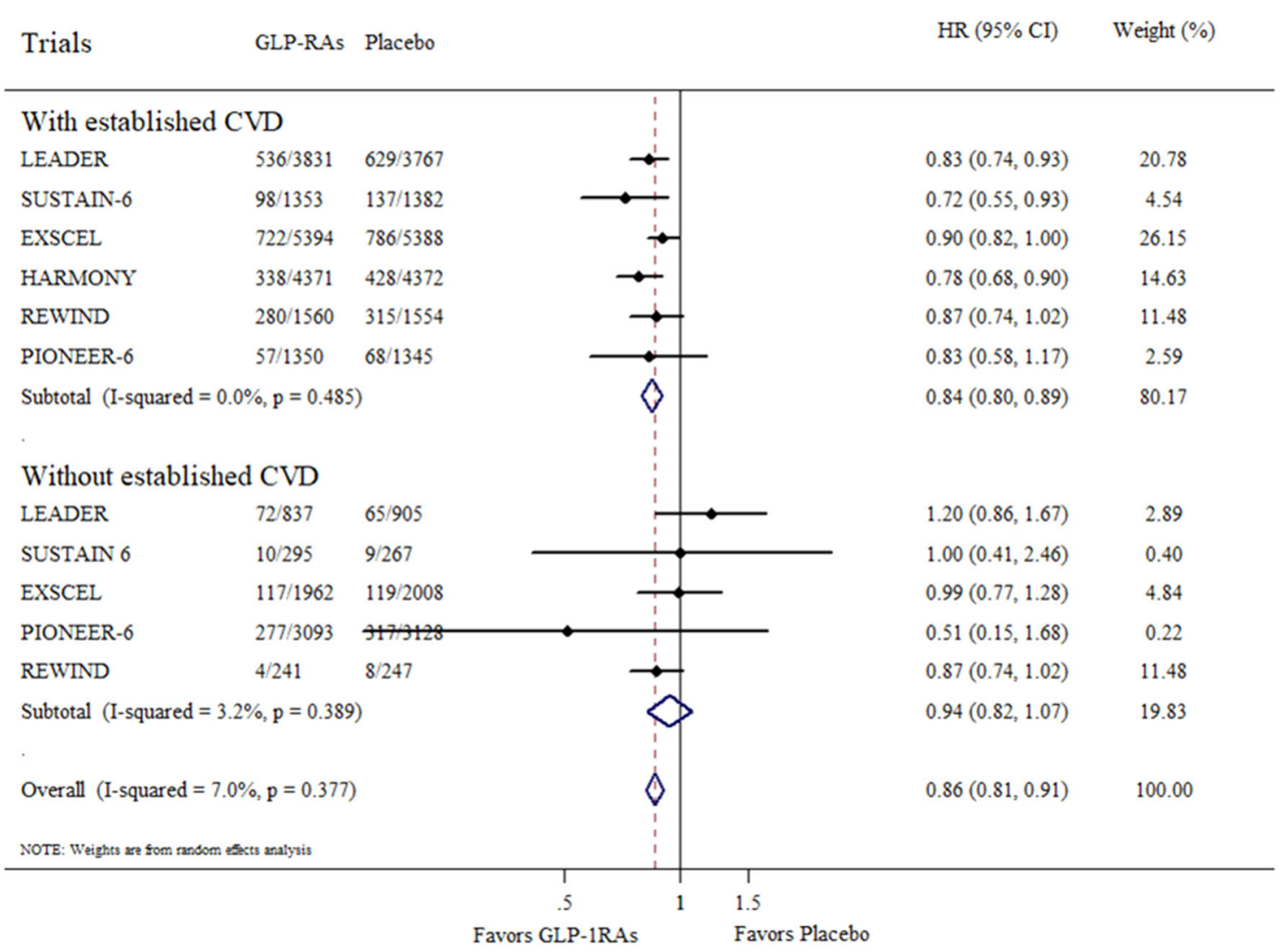

Fig. 3 Long-acting GLP-1RAs on MACE in patients with type 2 diabetes according to cardiovascular disease status. HR hazard ratio, CI confidence interval, GLP-1RAs

cancer (OR 1.31; 95\% CI 0.79-2.17; $P=0.300$ ) with long-acting GLP-1RAs compared to placebo, with low-to-moderate heterogeneity observed between trials. Regardless of the evident increase in the risk of retinopathy in SUSTAIN-6 (semaglutide vs. placebo, $3 \%$ vs. $1.8 \%$, $P=0.02$ ), no significant increase in risk was found in the overall results of retinopathy (OR 1.09; 95\% CI 0.92-1.29; $P=0.316$ ). A significantly increased risk of serious gastrointestinal events (OR 1.37; 95\% CI 1.02-1.83; $P=0.037$ ) and AEs leading to drug discontinuation (OR 1.38; 95\% CI 1.01-1.88; $P=0.039$ ) were observed, both showing consistent trends (Fig. 6). As shown in Fig. 7, there were no significant differences in SAEs between long-acting GLP-1RAs and placebo (OR 0.92; 95\% CI 0.85-1.00; $\quad P=0.039)$. The individual glucagon-like peptide 1 receptor agonists, CVD cardiovascular disease, $M A C E$ major adverse cardiovascular events

occurrence of SAEs by systemic organ varied, with no significant increase in risk, with the exception of serious gastrointestinal events (Table S5).

\section{DISCUSSION}

This systematic review and meta-analysis explored the influential factors and subpopulation differences associated with the cardiovascular efficacy of long-acting GLP-1RAs for patients with T2DM using six recent CVOTs. Among various subpopulations separately stratified by ten factors, there were no significant differences in the cardiovascular efficacy of long-acting GLP-1RAs. There was a favorable trend in the subpopulation with established 
Race

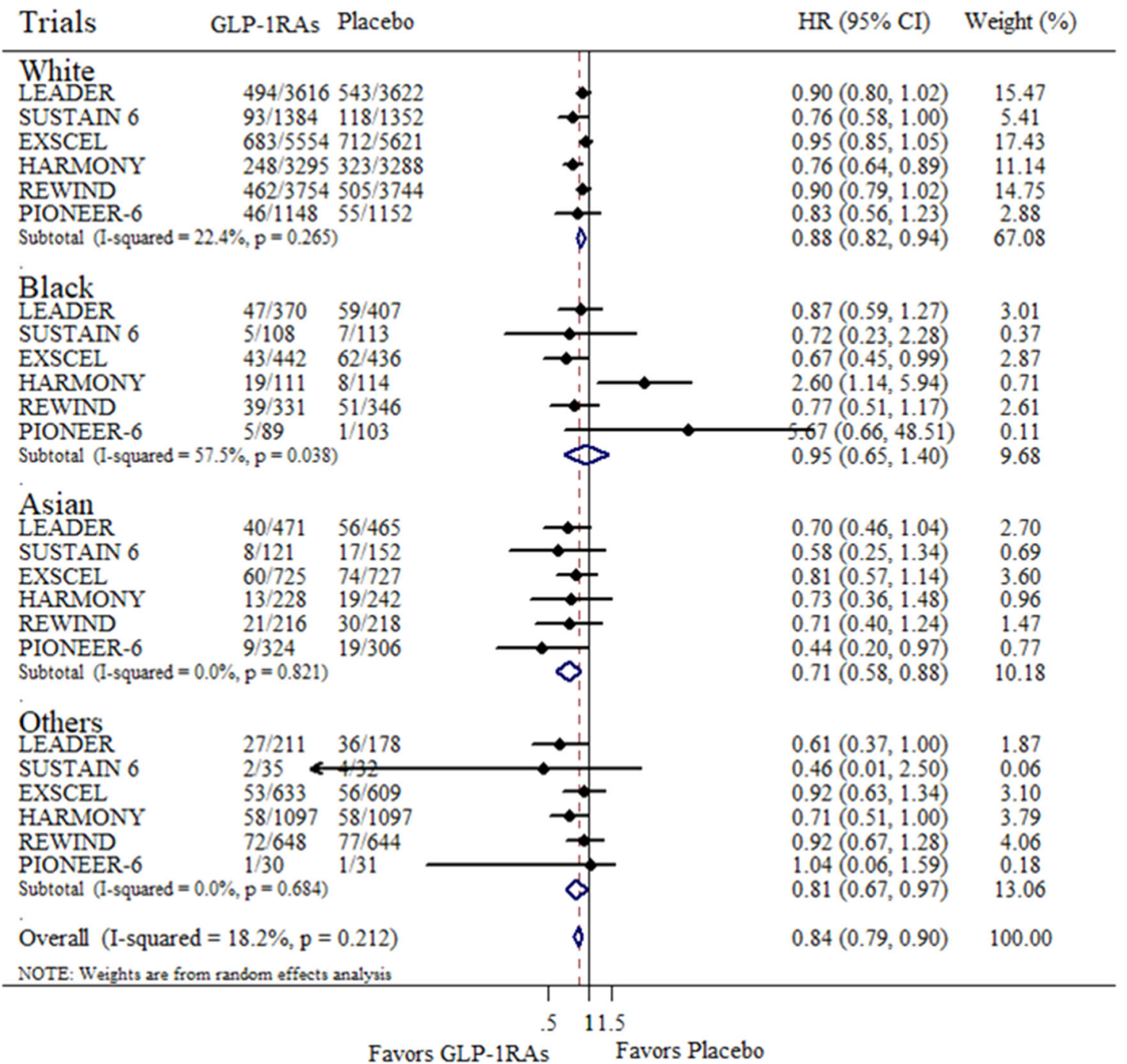

Fig. 4 Long-acting GLP-1RAs on MACE in patients with type 2 diabetes according to race. $H R$ hazard ratio, $C I$ confidence interval, GLP-1RAs glucagon-like peptide 1 receptor agonists, $M A C E$ major adverse cardiovascular events

CVD compared to those without established CVD. In addition to remarkable cardiovascular efficacy, treatment with long-acting GLP-1RAs achieved satisfactory safety profiles.

Among the included trials, only the PIONEER trial showed comparative risk reductions in patients without established CVD [15], whereas there were favorable trends towards achieving greater cardiovascular efficacy in the subpopulation with established CVD. However, the $P$ value for interaction regarding CVD status was insignificant. Similarly, there were no significant differences between subpopulations with different categories of baseline BMI. Since weight loss contributes to a reduced risk of CVD, and since GLP-1RAs can effectively promote weight loss, the subpopulation with a high baseline BMI was expected to have favorable effects over that with low baseline BMI. Previous studies regarding treatments with liraglutide reported greater effects of weight loss in subpopulations characterized by a high 


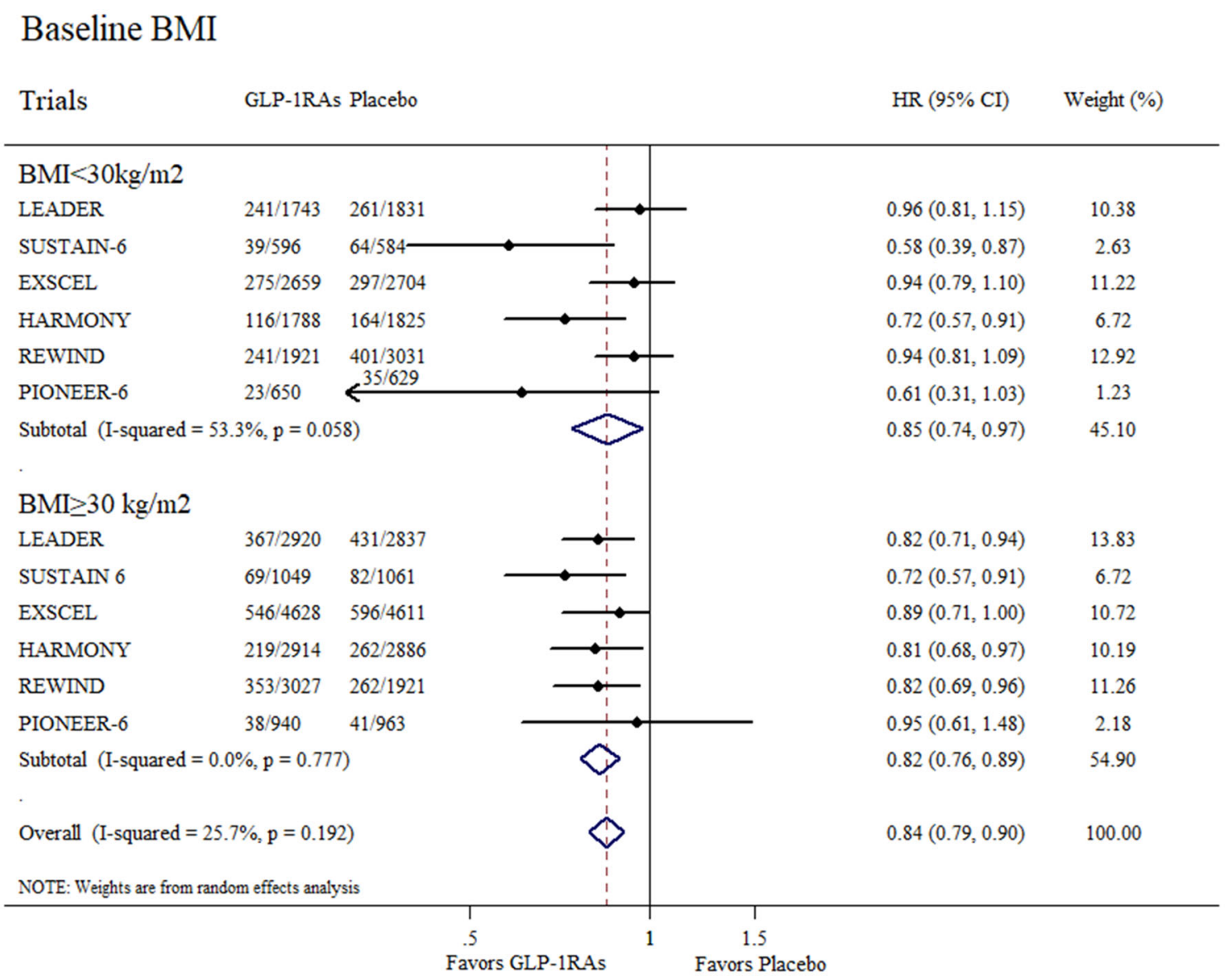

Fig. 5 Long-acting GLP-1RAs on MACE in patients with type 2 diabetes according to baseline BMI. HR hazard ratio, $C I$ confidence interval, GLP-1RAs glucagon-like peptide 1 receptor agonists, $B M I$ body mass index, $M A C E$ major adverse cardiovascular events

long-acting GLP-1RAs, while some studies described divergent results $[17,18]$. Despite ameliorative cardiac function in patients with T2DM and HF observed in previous nonrandomized pilot studies $[19,20]$, disappointing effects of GLP-1RA on HF have been reported in recent RCTs $[21,22]$. In light of previous studies, GLP-1 therapy might ameliorate advanced HF $[23,24]$. Conversely, liraglutide showed no greater benefit in patients with advanced HF in the FIGHT trial [22], and even increased the relative risk of $\mathrm{HF}$ readmission. Additionally, according to the LIVE sub-study liraglutide had no obvious effect on myocardial glucose uptake, myocardial blood flow, or myocardial blood flow reserve in patients with chronic HF without diabetes [25]. The exact relationship between long-acting GLP-1RAs and HF requires 


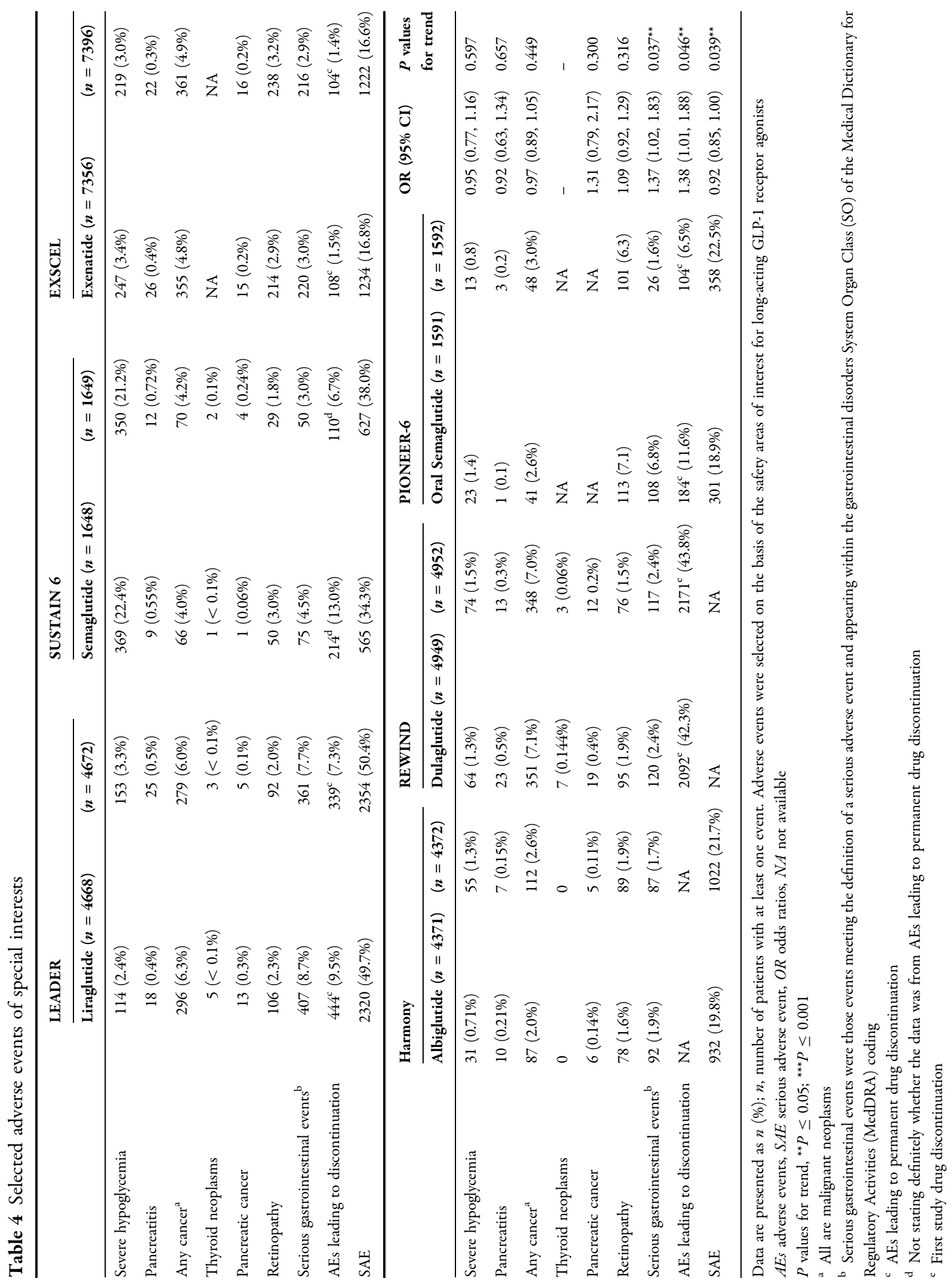




\section{Serious Gastrointestinal events}

\begin{tabular}{|c|c|c|c|c|}
\hline Trials & GLP-1RAs & Placebo & $\mathrm{OR}(95 \% \mathrm{CI})$ & Weight (\%) \\
\hline LEADER & 407 & 361 & $1.14(0.98,1.32)$ & 18.90 \\
\hline SUSTAIN 6 & 75 & 50 & $1.52(1.06,2.20)$ & 15.20 \\
\hline EXSCEL & 220 & 216 & $1.02(0.85,1.24)$ & 18.32 \\
\hline HARMONY OUTCOMES & 92 & 87 & $1.06(0.79,1.42)$ & 16.52 \\
\hline REWIND & 120 & 117 & $1.03(0.79,1.33)$ & 17.22 \\
\hline PIONEER-6 & 108 & 26 & $4.39(2.84,6.77)$ & 13.85 \\
\hline \multicolumn{3}{|c|}{ Overall (I-squared $=87.9 \%, p=0.000)$} & $1.37(1.02,1.83)$ & 100.00 \\
\hline NOTE: Weights se fom random escets an & & & & \\
\hline
\end{tabular}

\section{AEs leading to discontinuation}

Trials

GLP-1RAs Placebo

OR $(95 \% \mathrm{CI})$

Weight $(\%)$

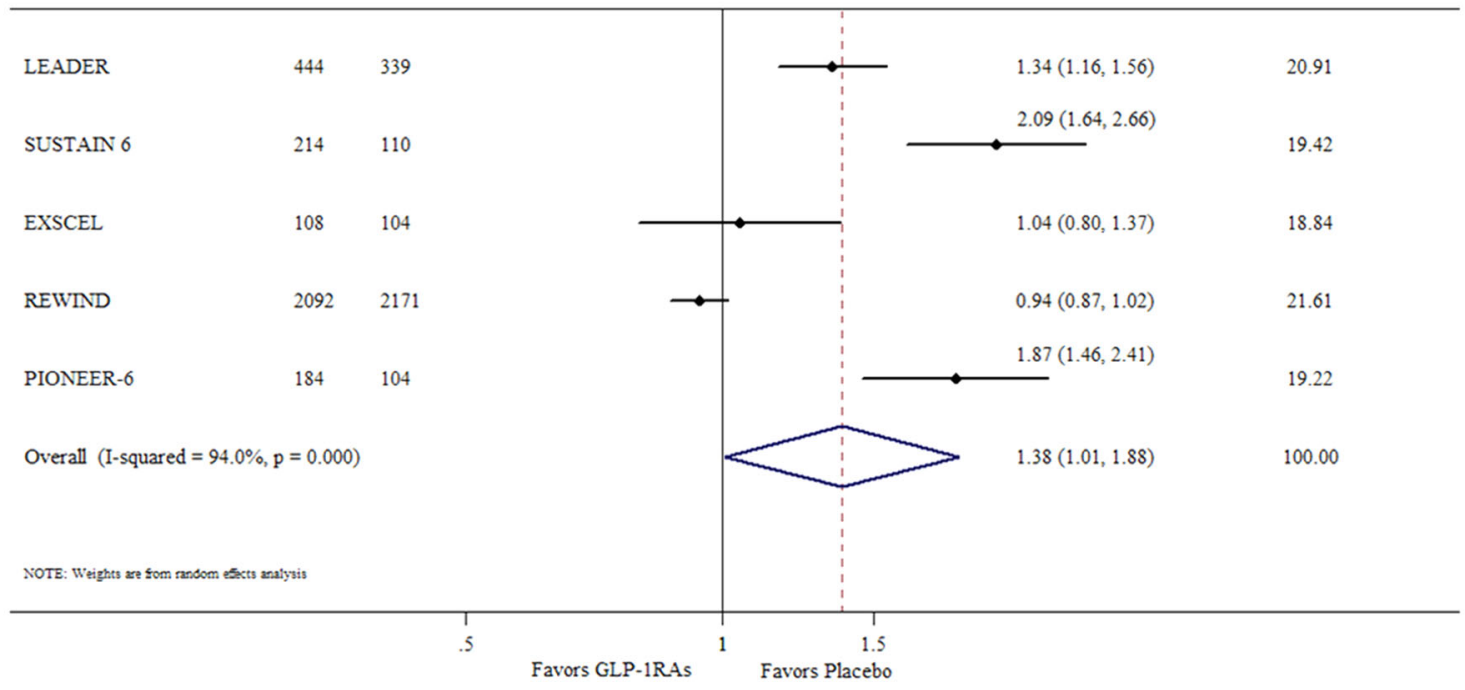

Fig. 6 Forest plot of serious gastrointestinal events and AEs leading to discontinuation. $O R$ odds ratio, $C I$ confidence interval, GLP-1RAs glucagon-like peptide 1 receptor agonists, $A E s$ adverse events 


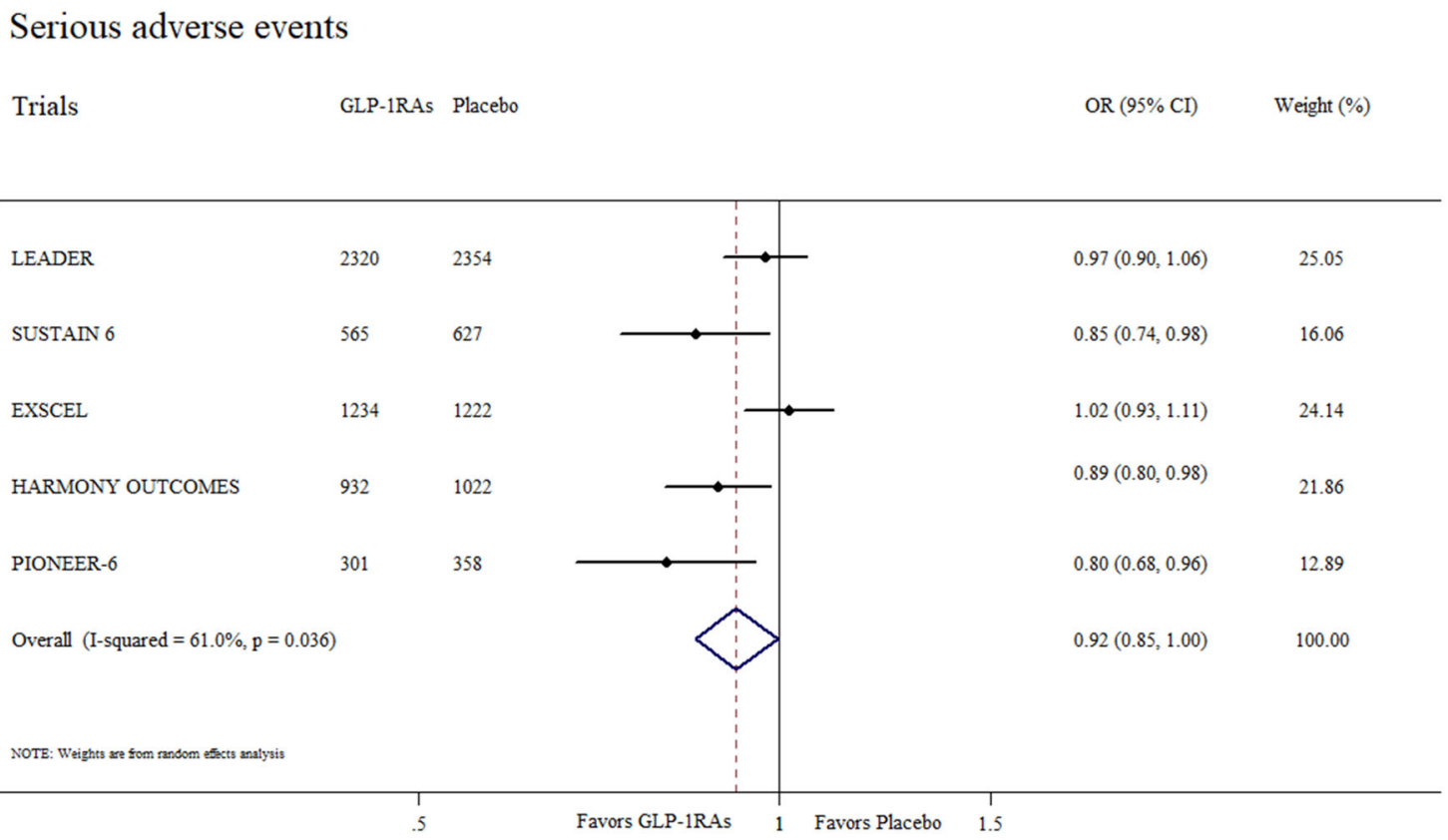

Fig. 7 Forest plot of serious adverse events. OR odds ratio, $C I$ confidence interval, GLP-1RAs glucagon-like peptide 1 receptor agonists

more highly qualified trials for further exploration.

Overall, long-acting GLP-1RAs are well tolerated and are generally safe, regardless of the increased risk of serious gastrointestinal events, which are generally controllable and manageable [26]. The overall results of AEs leading to drug discontinuation were similar to serious gastrointestinal events (Fig. 6), which implied that drug discontinuation was mainly due to serious gastrointestinal events. However, it remains unknown whether semaglutide specifically increases the risk of retinopathy. A recent study reported that the elevated risk of retinopathy was largely dependent on the baseline presence of pre-existing retinopathy, and that the occurrence of retinopathy primarily resulted from the magnitude and rapidity of glycemic reduction [27]. Furthermore, several previous studies reported no association between GLP-1R agonists and diabetic retinopathy [28-30], which is in agreement with the results of the current study.

Among numerous CVOTs, only seven trials have been completed: one trial of short-acting GLP-1Ra (ELIXA) and six trials of long-acting
GLP-1RAs (EXSCEL, LEADER, SUSTAIN-6, Harmony Outcomes, REWIND, and PIONEER-6). Several previous meta-analyses $[17,18]$ have elucidated favorable cardiovascular efficacy of GLP-1RAs without the inclusion of the more recent trials, REWIND and PIONEER-6. A recent updated meta-analysis [31] of GLP-RAs that was published as a brief report simply described the overall cardiovascular efficacy, but did not comprehensively explore the safety endpoints, or the influential factors and subpopulation differences of cardiovascular efficacy. Therefore, focusing on long-acting GLP-1RAs, the present study made comprehensive explorations of influential factors and subpopulation differences in cardiovascular efficacy of long-acting GLP-1RAs, as well as further analysis of the safety profile of GLP-1RAs. Giugliano and colleagues [31] reported that GLP-1RAs reduced three-point MACE by $13 \%$, with seven CVOTs of GLP-1RAs, the results of which supported those of the present study.

Nevertheless, there are some limitations in our study. Firstly, head-to-head trials were lacking, and each trial was conducted with different GLP-RAs. Different GLP-1RAs with varied 
properties might have different degrees of cardioprotective efficacy, which would weaken the reliability of the final conclusions to some extent. Secondly, the subjects recruited in each trial were mainly of white race and from Western countries, with very few Asian subjects. Thirdly, the stratifications of various subpopulations in different trials were not completely consistent. For example, the LEADER trial divided age as $<60$ years and $\geq 60$ years, while other trials chose 65 years as cutoff. Lastly, the duration of the trials might be a potential limitation.

\section{CONCLUSION}

There were no significant subpopulation differences in the cardiovascular benefits of longacting GLP-1RA treatments across various factors. These findings indicate that the administration of long-acting GLP-1RAs is unlikely to differ significantly among T2DM populations with different characteristics. Furthermore, the subpopulation with established CVD showed favorable trends over those without established CVD, which implies a possible propensity to utilize long-acting GLP-1RAs. With remarkable cardiovascular benefits for patients with T2DM, long-acting GLP-1RAs did not significantly increase the risk of retinopathy and SAEs overall. Our findings have implications in the management of long-acting GLP-1RA treatments across various subpopulations of patients with T2DM, and are of clinical importance in the safety of long-acting GLP-1RAs. Further large-scale studies that focus on the exploration of subpopulation differences with respect to the cardiovascular benefits of long-acting GLP-1RAs are required to verify our findings.

\section{ACKNOWLEDGEMENTS}

Funding. The current work was supported in part by the National Natural Science Foundation of China (91846106), the Non-profit Central Research Institute Fund of Chinese Academy of Medical Sciences Clinical, and the
Translational Medicine Research Fund (2019XK320029). The Chinese Academy of Medical Sciences Innovation Fund for Medical Sciences (CIFMS2016-I2M-4-001), the Training Program for Excellent Talents in Dongcheng District, and the Education Reforming Program, Peking Union Medical College (no. 2018zlgc0119) also funded the research. These organizations had no role in the design and conduct of the study; collection, management, analysis, and interpretation of the data; preparation, review, or approval of the manuscript; or the decision to submit the manuscript for publication. The journal's Rapid Service Fee was funded by the authors.

Authorship. All named authors meet the International Committee of Medical Journal Editors (ICMJE) criteria for authorship for this article, take responsibility for the integrity of the work as a whole, and have given their approval for this version to be published.

Authorship Contributions. Study concept and design: Huabing Zhang, Liyun $\mathrm{He}$, and $\mathrm{Na}$ Yang. Search of the relevant database and study identification: Liyun He, Huabing Zhang, and Lingling $\mathrm{Xu}$. Study selection: Liyun $\mathrm{He}, \mathrm{Na}$ Yang, and Fan Ping. Data extraction: Liyun He, Huabing Zhang, and Wei Li. Quality assessments of the eligible studies: Liyun He, Huabing Zhang, and Yuxiu Li. Confirmation of statistical analysis: Liyun He, Huabing Zhang, and Wei Li. Statistical analysis: LH and HZ. Examination for the methodology: LH, HZ, and FP. Drafting of the manuscript: Liyun He and Huabing Zhang. Critical revision of the manuscript for important intellectual content: Liyun He, Fan Ping, Wei Li, Lingling Xu, Huabing Zhang, and Yuxiu Li. Obtained funding: Huabing Zhang. Administrative, technical, and material support: Huabing Zhang. Study supervision: Huabing Zhang. All authors have approved the final article.

Disclosure. Liyun $\mathrm{He}, \mathrm{Na}$ Yang, Lingling $\mathrm{Xu}$, Fan Ping, Wei Li, Qi Sun, Yuxiu Li, and Huabing Zhang have nothing to disclose. 
Compliance with Ethics Guidelines. This article is based on previously conducted studies and does not contain any studies with human participants or animals performed by any of the authors.

Data Availability. The datasets generated during and/or analyzed during the current study are available from the corresponding author (Zhang) on reasonable request.

Open Access. This article is licensed under a Creative Commons Attribution-NonCommercial 4.0 International License, which permits any non-commercial use, sharing, adaptation, distribution and reproduction in any medium or format, as long as you give appropriate credit to the original author(s) and the source, provide a link to the Creative Commons licence, and indicate if changes were made. The images or other third party material in this article are included in the article's Creative Commons licence, unless indicated otherwise in a credit line to the material. If material is not included in the article's Creative Commons licence and your intended use is not permitted by statutory regulation or exceeds the permitted use, you will need to obtain permission directly from the copyright holder. To view a copy of this licence, visit http://creativecommons.org/licenses/by$\mathrm{nc} / 4.0 /$.

\section{REFERENCES}

1. American Diabetes Association, the National Heart, Lung, and Blood Institute, the Juvenile Diabetes Foundation International, the National Institute of Diabetes and Digestive and Kidney Diseases, the American Heart Association. Diabetes mellitus: a major risk factor for cardiovascular disease. Circulation. 1999;100(10):1132-3. https://doi.org/10. 1161/01.cir.100.10.1132.

2. Drucker DJ. Mechanisms of action and therapeutic application of glucagon-like peptide-1. Cell Metab. 2018;27(4):740-56. https://doi.org/10.1016/j.cmet. 2018.03.001.

3. Drucker DJ. The ascending GLP-1 road from clinical safety to reduction of cardiovascular complications.
Diabetes. 2018;67(9):1710-9. https://doi.org/10. 2337/dbi18-0008.

4. Das SR, Everett BM, Birtcher KK, et al. 2018 ACC expert consensus decision pathway on novel therapies for cardiovascular risk reduction in patients with type 2 diabetes and atherosclerotic cardiovascular disease: a report of the American College of Cardiology task force on expert consensus decision pathways. J Am Coll Cardiol. 2018;72(24):3200-23. https://doi.org/10.1016/j.jacc.2018.09.020.

5. Pfeffer MA, Claggett B, Diaz R, et al. Lixisenatide in patients with type 2 diabetes and acute coronary syndrome. N Engl J Med. 2015;373(23):2247-57.

6. Davies MJ, D’Alessio DA, Fradkin J, et al. Management of hyperglycemia in type 2 Diabetes, 2018. A consensus report by the American Diabetes Association (ADA) and the European Association for the Study of Diabetes (EASD). Diabetes Care. 2018;41(12):2669-701. https://doi.org/10.2337/ dci18-0033.

7. Zelniker TA, Wiviott SD, Raz I, et al. SGLT2 inhibitors for primary and secondary prevention of cardiovascular and renal outcomes in type 2 diabetes: a systematic review and meta-analysis of cardiovascular outcome trials. Lancet. 2019;393(10166):31-9. https://doi.org/10.1016/ s0140-6736(18)32590-x.

8. Stewart LA, Clarke M, Rovers M, et al. Preferred reporting items for systematic review and metaanalyses of individual participant data: the PRISMAIPD statement. JAMA. 2015;313(16):1657-65. https://doi.org/10.1001/jama.2015.3656.

9. Higgins JP, Altman DG, Gotzsche PC, et al. The Cochrane Collaboration's tool for assessing risk of bias in randomised trials. BMJ. 2011;343:d5928. https://doi.org/10.1136/bmj.d5928.

10. Marso SP, Daniels GH, Brown-Frandsen K, et al. Liraglutide and cardiovascular outcomes in type 2 diabetes. N Engl J Med. 2016;375(4):311-22. https://doi.org/10.1056/NEJMoa1603827.

11. Marso SP, Bain SC, Consoli A, et al. Semaglutide and cardiovascular outcomes in patients with type 2 diabetes. N Engl J Med. 2016;375(19): $1834-44$.

12. Holman RR, Bethel MA, Mentz RJ, et al. Effects of once-weekly exenatide on cardiovascular outcomes in type 2 diabetes. $\mathrm{N}$ Engl J Med. 2017;377(13): 1228-399. NEJMoa1612917.

13. Hernandez AF, Green JB, Janmohamed S, et al. Albiglutide and cardiovascular outcomes in patients with type 2 diabetes and cardiovascular disease 
(Harmony Outcomes): a double-blind, randomised placebo-controlled trial. Lancet. 2018;392(10157): 1519-29. https://doi.org/10.1016/s01406736(18)32261-x.

14. Gerstein HC, Colhoun HM, Dagenais GR, Diaz R, Lakshmanan M, Pais P, et al. Dulaglutide and cardiovascular outcomes in type 2 diabetes (REWIND): a double-blind, randomised placebo-controlled trial. Lancet. 2019. https://doi.org/10.1016/s01406736(19)31149-3.

15. Husain M, Birkenfeld AL, Donsmark M, et al. Oral semaglutide and cardiovascular outcomes in patients with type 2 diabetes. New Engl J Med. 2019;381:841-51.

16. Niswender K, Pi-Sunyer X, Buse J, et al. Weight change with liraglutide and comparator therapies: an analysis of seven phase 3 trials from the liraglutide diabetes development programme. Diabetes Obes Metab. 2013;15(1):42-544. https://doi. org/10.1111/j.1463-1326.2012.01673.x.

17. Kang YM, Cho YK, Lee J, et al. Asian subpopulations may exhibit greater cardiovascular benefit from long-acting glucagon-like peptide 1 receptor agonists: a meta-analysis of cardiovascular outcome trials. Diabetes Metab J. 2018. https://doi.org/10. 4093/dmj.2018.0070.

18. Bethel MA, Patel RA, Merrill P, et al. Cardiovascular outcomes with glucagon-like peptide-1 receptor agonists in patients with type 2 diabetes: a metaanalysis. Lancet Diabetes Endocrinol. 2018;6(2): 105-13.

19. Nikolaidis LA, Mankad S, Sokos GG, et al. Effects of glucagon-like peptide- 1 in patients with acute myocardial infarction and left ventricular dysfunction after successful reperfusion. Circulation. 2004;109(8):962-5.

20. Sokos GG, Nikolaidis LA, Mankad S, Elahi D, Shannon RP. Glucagon-like peptide-1 infusion improves left ventricular ejection fraction and functional status in patients with chronic heart failure. J Cardiac Fail. 2006;12(9):694-9.

21. Jorsal A, Kistorp C, Holmager P, et al. Effect of liraglutide, a glucagon-like peptide- 1 analogue, on left ventricular function in stable chronic heart failure patients with and without diabetes (LIVE) - a multicentre, double-blind, randomised, placebocontrolled trial. Eur J Heart Fail. 2017;19(1):69-77.

22. Margulies KB, Hernandez AF, Redfield MM, et al. Effects of liraglutide on clinical stability among patients with advanced heart failure and reduced ejection fraction: a randomized clinical trial. JAMA. 2016;316(5):500-8.

23. Nikolaidis LA, Sturzu A, Stolarski C, Elahi D, Shen Y-T, Shannon RP. The development of myocardial insulin resistance in conscious dogs with advanced dilated cardiomyopathy. Cardiovasc Res. 2004;61(2):297-306.

24. Swan JW, Anker SD, Walton C, et al. Insulin resistance in chronic heart failure: relation to severity and etiology of heart failure. J Am Coll Cardiol. $1997 ; 30(2): 527-32$.

25. Nielsen R, Jorsal A, Iversen $P$, et al. Effect of liraglutide on myocardial glucose uptake and blood flow in stable chronic heart failure patients: a double-blind, randomized, placebo-controlled LIVE sub-study. J Nuclear Cardiol. 2019;26(2):585-97.

26. Gough SC. Liraglutide: from clinical trials to clinical practice. Diabetes Obes Metab. 2012;14(Suppl 2):33-40. https://doi.org/10.1111/j.1463-1326. 2012.01576.x.

27. Vilsboll T, Bain SC, Leiter LA, et al. Semaglutide, reduction in glycated haemoglobin and the risk of diabetic retinopathy. Diabetes Obes Metab. 2018;20(4):889-97. https://doi.org/10.1111/dom. 13172 .

28. Tang H, Li G, Zhao Y, et al. Comparisons of diabetic retinopathy events associated with glucose-lowering drugs in patients with type 2 diabetes mellitus: a network meta-analysis. Diabetes Obes Metab. 2018;20(5):1262-79. https://doi.org/10.1111/dom. 13232 .

29. Saw M, Wong VW, Ho IV, Liew G. New anti-hyperglycaemic agents for type 2 diabetes and their effects on diabetic retinopathy. Eye. 2019. https:// doi.org/10.1038/s41433-019-0494-z.

30. Wang T, Lu W, Tang H, Buse JB, Sturmer T, Gower EW. Assessing the association between GLP-1 receptor agonist use and diabetic retinopathy through the FDA adverse event reporting system. Diabetes Care. 2019;42(2):e21-e2323. https://doi. org/10.2337/dc18-1893.

31. Giugliano D, Maiorino MI, Bellastella G, Longo M, Chiodini P, Esposito K. GLP-1 receptor agonists for prevention of cardiorenal outcomes in type 2 diabetes: an updated meta-analysis including the REWIND and PIONEER 6 trials. Diabetes Obes Metab. 2019. https://doi.org/10.1111/dom.13847. 